\title{
First neutron spectrometry measurements in the ASDEX Up- grade tokamak
}

G. Tardini ${ }^{1}$, A. Zimbal ${ }^{2}$, B. Esposito ${ }^{3}$, F. Gagnon-Moisan ${ }^{2}$, D. Marocco ${ }^{3}$, R. $\mathrm{Neu}^{1}, \mathrm{H}$. Schuhmacher ${ }^{2}$, and the ASDEX Upgrade Team ${ }^{1}$

${ }^{1}$ MPI für Plasmaphysik, Euratom Association, Boltzmannstr. 2, 85748 Garching, Germany

2 Physikalisch-Technische Bundesanstalt, Bundesallee 100, 38116 Braunschweig, Germany

${ }^{3}$ Euratom-ENEA Association, Via E. Fermi 45, 00044 Frascati, Roma, Italy

\begin{abstract}
A compact neutron spectrometer based on the liquid scintillator BC501A has been installed on the ASDEX Upgrade tokamak. The aim is to measure neutron energy distribution functions as footprints of fast ions distribution functions, generated mainly via Neutral Beam Injection (NBI) in present day tokamaks. A flexible and fast software has been developed to perform digital pulse shape separation and to evaluate pulse height spectra. First measurements of count rates and pulse height spectra show a good signal to noise ratio for integration times comparable to the NBI slowing down time and to the energy confinement time. Due to the perpendicular line of sight, D-d fusion with perpendicular NBI is detected more efficiently and the line broadening of the $2.45 \mathrm{MeV}$ neutrons is higher. Ion Cyclotron Resonance Heating (ICRH) combined to NBI exhibits a synergy effect, with count rates higher than the sum of the counts due to NBI and ICRH separately. Although the collimator is designed to screen gammas as much as possible, some qualitative gamma analysis is also possible, providing information in case of runaway electrons during disruptions. The experimental campaign for the characterisation of the system (detector + acquisition system) is complete and the determination of the response function is in progress.
\end{abstract}

\section{Introduction}

The dynamics of suprathermal ions in tokamak plasmas has received growing interest in the last decade due to their role in large devices such as ITER, where highly energetic fusion alpha particles have to be confined both to meet the performance requirements and to avoid a deleterious impact on the 
Plasma Facing Components. Fast ions are known to drive Alfven Eigenmodes and to extend the sawtooth period, thus making a single sawtooth crash more violent. On the other hand, most of the mentioned Magneto-HydroDynamic (MHD) modes expel fast ions from the plasma, as it has been both observed experimentally and predicted theoretically [1][2]. The assessment of the ion distribution function has therefore stimulated several new diagnostic systems on ASDEX Upgrade [3]. In Neutral Beam Injection (NBI) ASDEX Upgrade discharges most neutrons arise from beam-target fusion reactions [4]. As the neutron energy distribution function can be considered a footprint of the fast ion distribution function, a Compact Neutron Spectrometer (CNS) [5] developed at the Physikalisch-Technische Bundesanstalt (PTB) has been installed on ASDEX Upgrade [6]. The installation geometry and the hardware details of the spectrometer's components (detector, electronics and collimator) are presented in Section 2. The software tool and the settings for the Digital Pulsed Shape Discrimination (DPSD) [7][8][9] are presented in Section 3, with an assessment of the neutron-gamma discrimination efficiency. First physics results are then presented in Section 4, before the status of the diagnostic and future enhancements are discussed in Section 5.

\section{Geometry and hardware of the Compact Neutron Spectrometer}

The PTB CNS is placed outside the torus hall (Fig. 1) at roughly $10 \mathrm{~m}$ from the closest plasma surface. It is based on a cylindrical BC501A liquid scintillator cell $(50.8 \times 50.8 \mathrm{~mm})$, connected to a photomultiplier (PMT) through a truncated cone-shaped light guide. A Light Emitting Diode (LED) provides the PMT with a $100 \mathrm{~ns}$ long reference pulse every $1 \mathrm{~ms}$, in order to monitor the PMT gain, which is known to be sensitive to the count rate. Further technical details are described in reference [6]. The line of sight of the detector is shown in Fig. 1 (b). The $2 \mathrm{~m}$ thick concrete wall of the torus hall provides a collimation, as well as a significant reduction of any scattered and background radiation. The line of sight is $10 \mathrm{~cm}$ above the geometrical equatorial plane, which corresponds to the plasma midplane. It is almost perpendicular to the magnetic surfaces, therefore the detector collects only neutrons with perpendicular velocity with respect to the magnetic field lines. A pre-collimator (a polyethylene filled cube, each side is $60 \mathrm{~cm}$ long) with an 


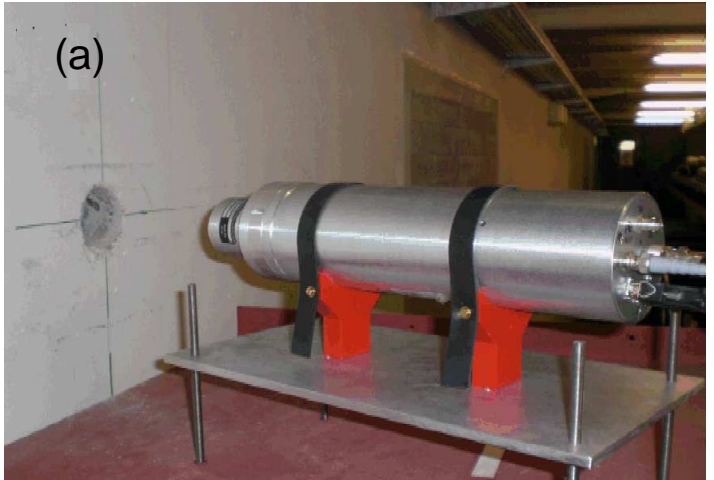

CNS location

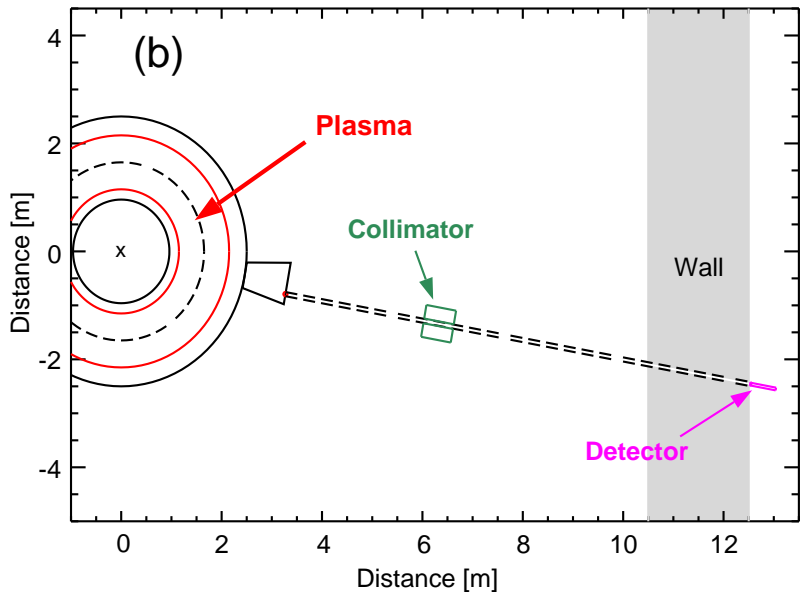

Figure 1: The CNS installed on the ASDEX Upgrade tokamak (a) and the system's geometry and line of sight (b). The red lines mark the plasma surface, at roughly $10 \mathrm{~m}$ distance from the detector.

open cylindrical cavity ( $88 \mathrm{~mm}$ diameter) improves the neutron collimation in the torus hall (Fig. 2). A $5 \mathrm{~cm}$ thick lead shield reduces the gamma flow

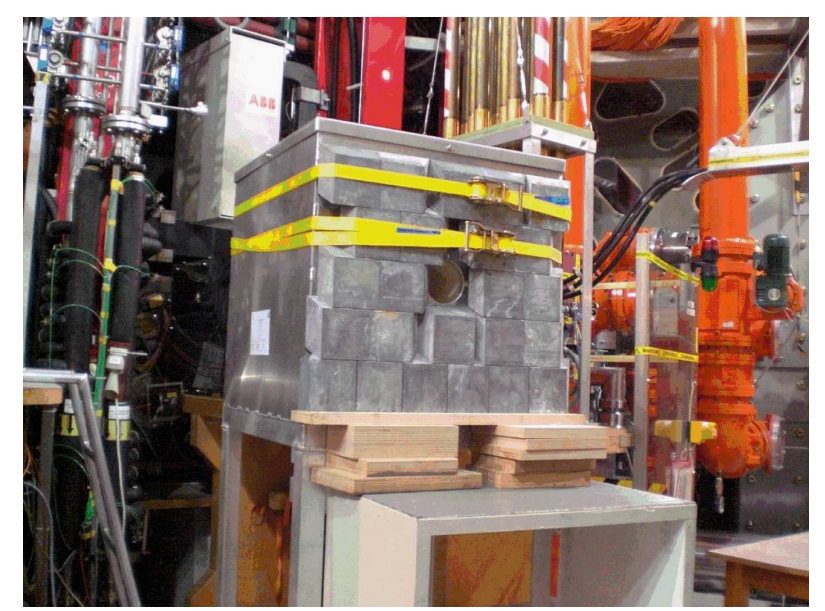

Figure 2: The polyethylene collimator in the torus hall

from the torus hall and the gammas produced in the pre-collimator by about $90 \%$, allowing higher neutron count rates.

The PMT is connected to a Digital Acquisition (DAQ) board developed at ENEA Frascati [7]. This board is equipped with two synchronised ADCs 
working at $100 \mathrm{MHz}$ rate each, which ensures a $5 \mathrm{~ns}$ sampling of the PMT signal. After the acquisition, the digital pulses are transferred to the disk. The board discards pulses below a pre-set threshold. For acquired pulses, though, the board does collect several buffered pre-trigger samples, which are important to determine and subtract the pulses' baseline. Moreover, a dynamic window length determination is adopted [8][10], to minimise the amount of samples required for each pulse and hence to enable new acquisitions as quickly as possible. The acquisition is limited in file size to 1.28 GBytes by the computer RAM and in data transmission to 80 MBytes/s (transfer from the board to the RAM of the acquisition PC via a PCI connection), the latter being the actual bottle neck. Considering the typical window length of $\approx 50$ samples $(250 \mathrm{~ns})$, the resulting saturation level for the count rate is:

$$
Y_{\text {sat }} \approx 80 \frac{\text { MBytes } / \mathrm{s}}{2 \text { Bytes } / \text { sample } * 50 \text { samples } / \text { pulse }}=0.8 \mathrm{Mpulses} / \mathrm{s}
$$

Should the transmission rate get improved by hardware enhancement, pile-up events should be included in the analysis. This possibility has been studied in [11] but it is not yet implemented in the present analysis tools. Pile-up events would become dominant above a few Mpulses/s .

The saturation limit described in equation 1 has been confirmed experimentally, as shown in Fig. 3, comparing a case above the limit (a) and one below (b). In the saturated case (Fig. 3 (a)), the board stops acquiring just about exceeding 1 Mpulses/s, consistently with the estimate 1 . When the rate drops below $1 \mathrm{Mpulses} / \mathrm{s}$ again, the acquisition restarts: the grey region in Fig. 3 indicates the time slice in which saturation occurs. When the rate is lower (Fig. 3 (b)), the time behaviour matches nicely the ASDEX Upgrade standard neutron monitor, following the quick variation due to the NBI power modulation.

\section{Digital Pulse Shape Discrimination}

Measurements of ASDEX Upgrade plasmas were taken routinely during the 2011 campaign, with fixed settings, in order to obtain deconvoluted spectra as soon as the response matrix will be evaluated from the data of the characterisation campaign, already performed at the PTB. Acquisitions last $120 \mathrm{~s}$, starting roughly $60 \mathrm{~s}$ before the plasma break-down. The hardware 

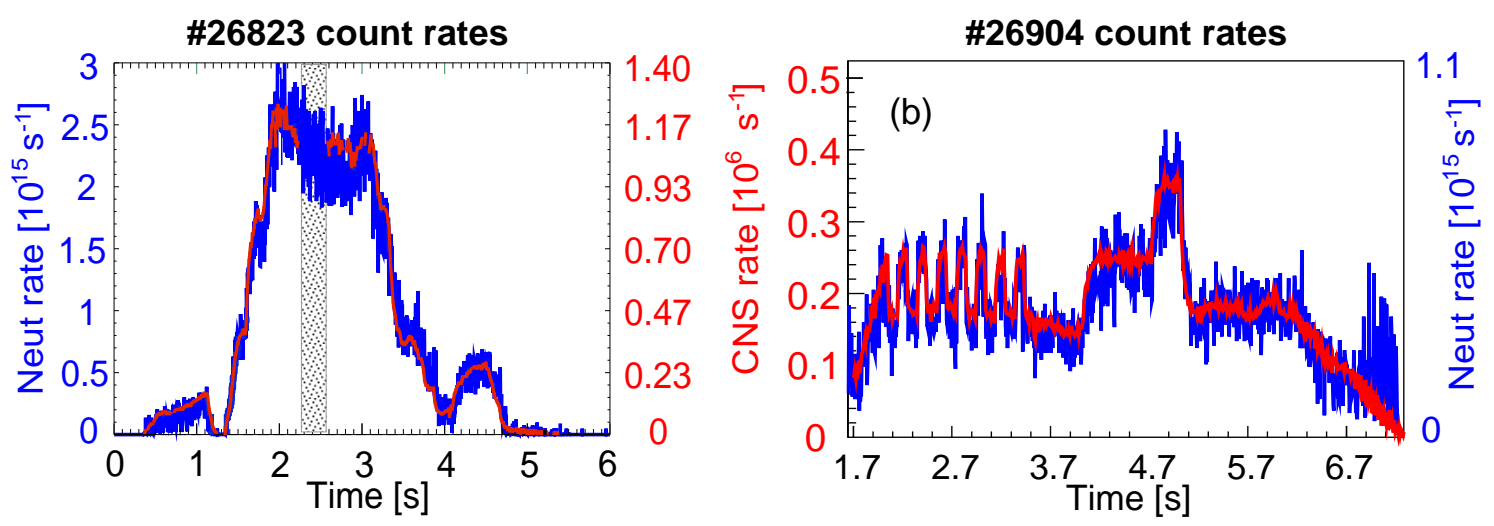

Figure 3: Comparison between the standard ASDEX Upgrade neutron monitor (blue) and the CNS (red) for a case with high neutron rate (a) and one with low rate (b). In the saturated case the DAQ time base has a gap (grey region).

acquisition threshold was set to $24 \mathrm{mV}$, corresponding to 64 channels. Eight pre-trigger samples determine the baseline of each single pulse, which is subtracted a posteriori for a correct pulse energy evaluation. The energy range has been optimised for neutron energies between 1.5 and $17 \mathrm{MeV}$, including both the D-d and d-t neutron distributions.

A software has been developed to compute and visualise the pulse shape as well as Pulse Height Spectra (PHS). The program is written in $\mathrm{C}++$, using the ROOT framework for the Graphic User Interface and analysis functions. The simple peak detection algorithm implemented so far [9] proves very fast and yet efficient, with only $5 \%$ of the pile-up events misinterpreted as single pulses, while the total amount of pile-ups is typically below $10 \%$ of the total count, depending on the count rate.

LED pulses are recognised and used for an off-line gain correction, since the PMT gain is sensitive to changes in the count rate. LED pulses are emitted every $1 \mathrm{~ms}$ and are averaged over $10 \mathrm{~ms}$ (10 pulses), a time interval shorter than the relevant plasma time scales, such as the slowing down time and the energy confinement time, both ranging between 20 and $50 \mathrm{~ms}$. The remaining single pulses are then separated into gammas and neutrons, exploiting the different decay time of the PMT signal of the different detected secondary charged particles [12]. As a figure of merit for the pulse shape we choose the ratio of short to long gate, $G R=Q_{S} /\left(Q_{L}-Q_{S}\right)$. In the optimised setup, 
the short gate is the pulse integral over the 3 samples following the peak, while the long gate is integrated over 14 samples after the peak, as shown in Fig. 4. The "total integral" is the numerical integral of the pulse over its whole time window. Fig. 5 shows a typical n-gamma PSD obtained with

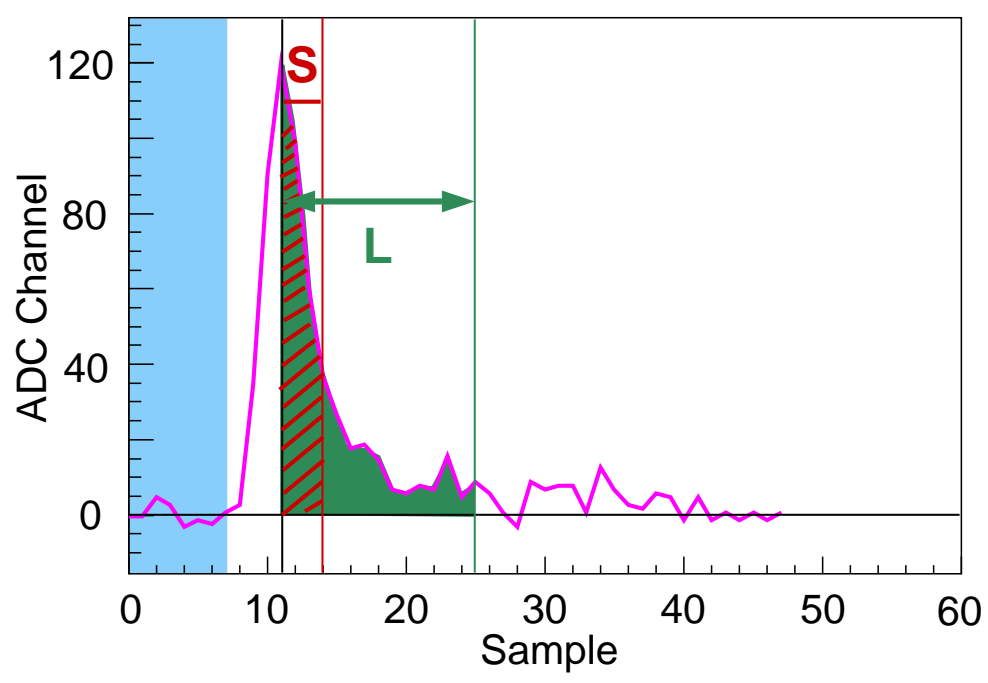

Figure 4: Typical single pulse used as input of the discrimination algorithm. The short gate $Q_{S}$ corresponds to the red dashed area, the long gate $Q_{L}$ to the green area. The blue area marks the pre-trigger region.

this board. The separation in terms of the ratio $G R$ turns out to be efficient. This means that the detector is suited for pulse shape discrimination, and also that the digital time resolution is sufficiently high. A two-slope line is used to discriminate gammas from neutrons. In the same Fig. 5, the LED pulses can be easily recognised, since their intensity is optimised to be still inside the corresponding relevant range of proton energy, but well apart from the measured signal. Finally, figure 5 shows also the presence of some burn-up d-t neutrons.

\section{$4 \quad$ First physics results}

Although the final goal is to obtain the deconvoluted energy distribution function, some useful information can be drawn already looking at the count rates and at the PHS. A first test of the physics soundness of the CNS measurements is the selectivity in the neutron velocity direction. Most neutrons 


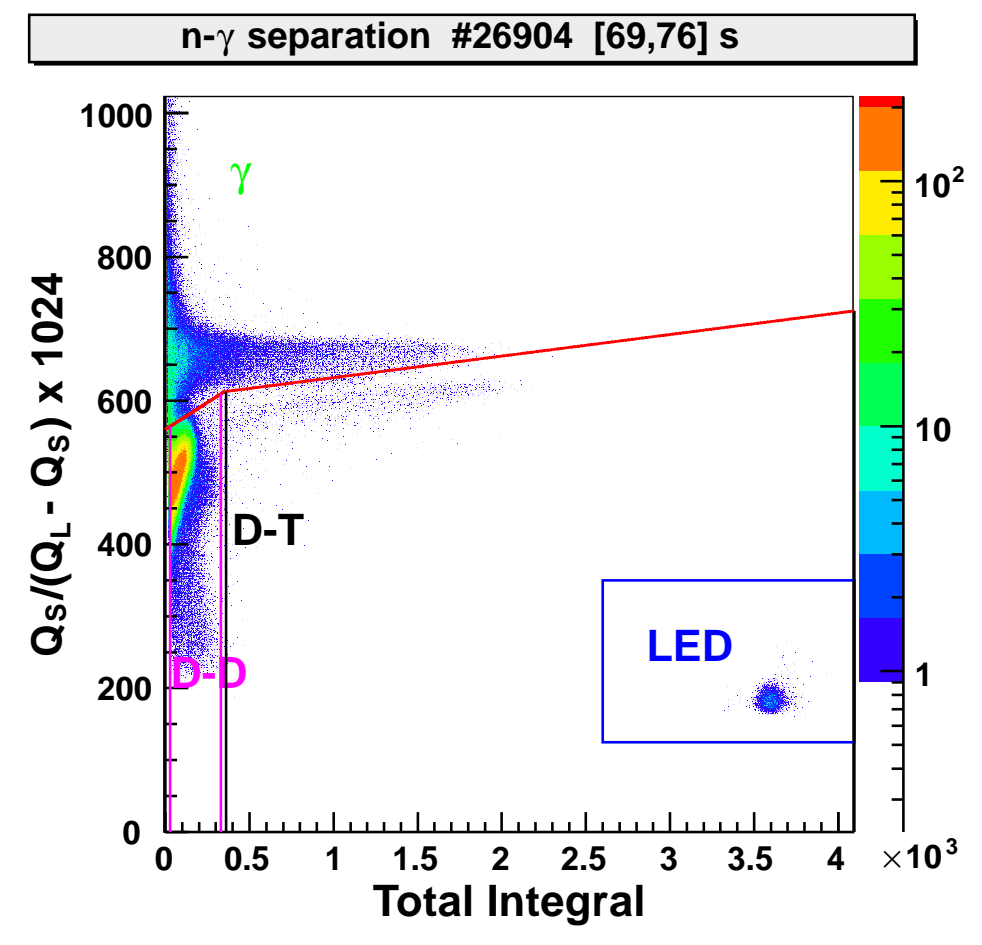

Figure 5: Gate ratio $G R$ as a function of the total pulse integral. The reference LED pulses are well separated. d-t burn-up neutrons are detected as well.

in ASDEX Upgrade come from beam-target D-d fusion reactions [4], the beam being provided by the NBI. The flexible geometry of the NBI systems allows both perpendicular and parallel injection with respect to the magnetic field. In case of perpendicular injection, from simple kinematics the outcoming neutrons are expected to be detected with a higher intensity and with a wider broadening of their distribution, due to the perpendicular line of sight of the CNS. In discharge \# 26939, shown in Fig. 6, there are 3 phases: first perpendicular NBI, then parallel, then perpendicular again. Comparing the CNS counts time trace with the reference ${ }^{238} \mathrm{U}$ fission chamber normalised to the CNS signal in the first phase (perpendicular NBI), it appears that switching to parallel NBI reduces both signals, but the relative decrease of the CNS count rate is significantly higher than the one of the reference neutron counter. This is due to the beam-target reaction kinematics, which in the laboratory frame is anisotropic, with a preferencial 


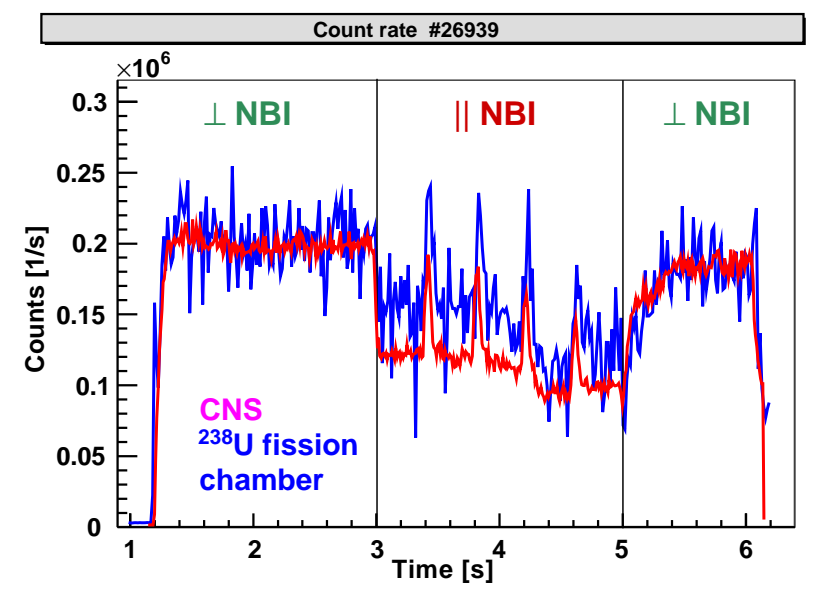

Figure 6: Count rate as a function of time for discharge \# 26939, in 3 phases: perpendicular NBI, parallel NBI, perpendicular NBI. CNS (red) compared to the reference neutron monitor (blue) normalised to the CNS signal during the first perpendicular phase. 4 NBI blips are added in the parallel NBI phase, for other measurements.

direction when the beam ion is directed along the collimator line of sight, i.e. in our case perpendicular to the magnetic surfaces. As expected, switching back to perpendicular NBI, the two signals fall again on top of each other. Also the normalised PHS exhibit the expected behaviour, plotted in Fig. 7. Normalising the PHS corresponding to perpendicular NBI (green line in Fig. 7) to the PHS associated with parallel NBI (red curve) at channels 50-100, the broader PHS for the case with perpendicular NBI is highlighted. This is consistent with the reaction's kinematics in the laboratory frame.

One of the most interesting applications of the CNS to ASDEX Upgrade plasmas is the investigation of the synergy effect between NBI and the Ion Cyclotron Resonance Heating [13][14]. It is a common observation in tokamaks that adding radio-frequency heating on top of NBI enhances the neutron rate by more than the rate associated with ICRH only under the same experimental conditions and plasma parameters [15]. An example for an ASDEX Upgrade discharge is shown in Fig. 8. In discharge \# 26998 there is first a phase with pure NBI heating $(5 \mathrm{MW})$. After $3.5 \mathrm{~s}, P_{N B I}$ is reduced to $2.5 \mathrm{MW}$ and the CNS count rate (magenta curve) drops from $1.610^{5}$ to $1.110^{5}$ counts/s (Fig. 8 a)). At the same time, the ICRH is ramped up to $P_{I C R H}=5 \mathrm{MW}$, enhancing the neutron count by $0.610^{5}$ counts/s gradually. 


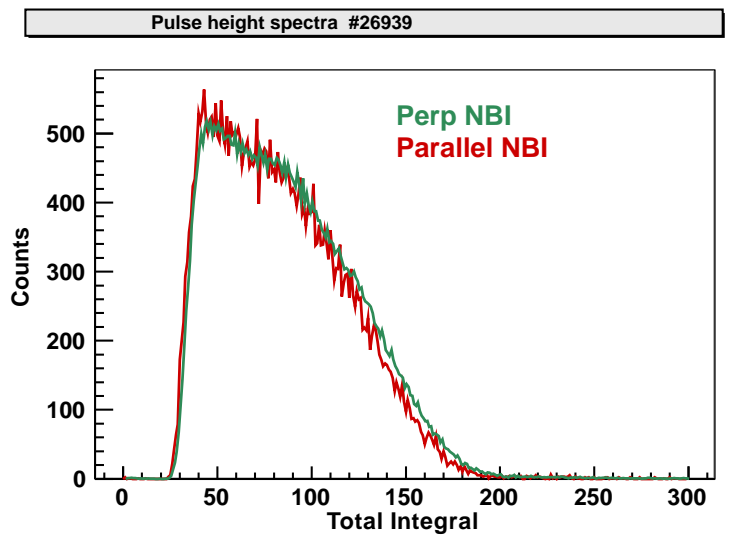

Figure 7: PHS for discharge \# 26939 for perpendicular (green) and parallel NBI (red). Broader PHS are observed in the phase with perpendicular NBI.
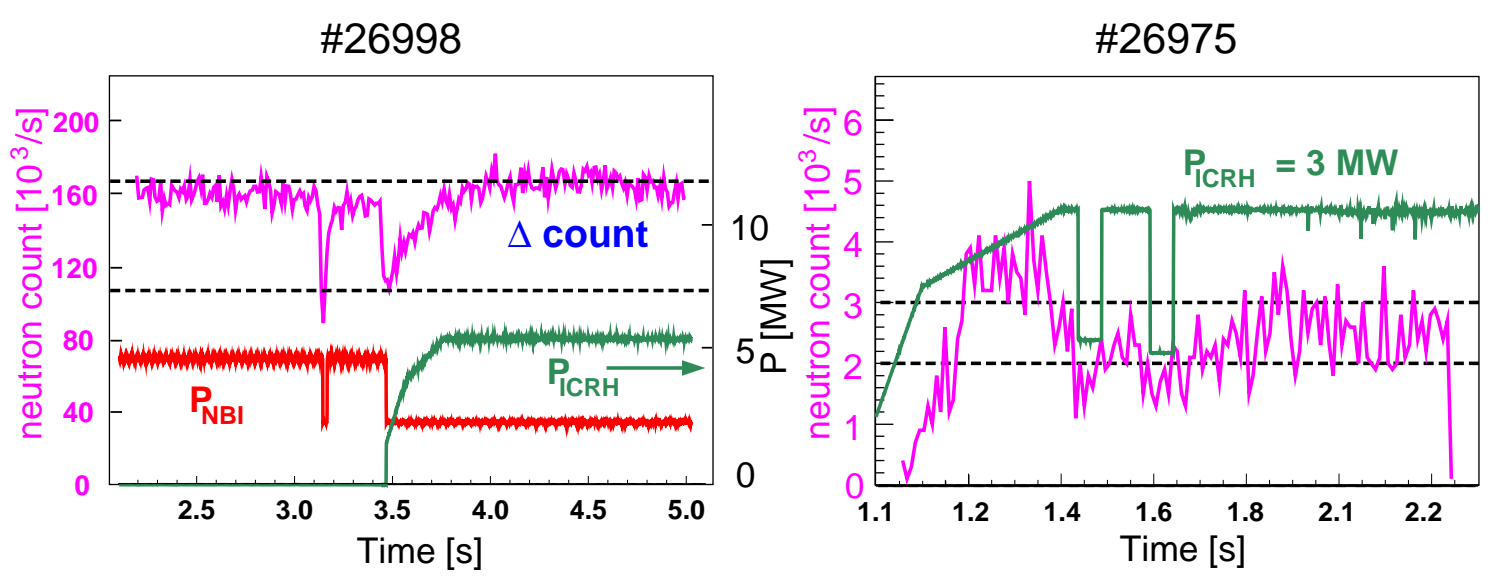

Figure 8: Neutron count rates for discharge \# 26998 in a phase with $P_{N B I}=5 \mathrm{MW}, P_{I C R H}=0$ and in a phase with $P_{N B I}=2.5 \mathrm{MW}, P_{I C R H}=5 \mathrm{MW}$ (green).

For comparison, discharge \# 26975 with pure ICRH (3 MW) is shown in Fig. 8 b). There, the CNS count rate varies between 2 and $310^{3}$ : so, even taking into account the lower $P_{I C R H}$, this more than one order of magnitude smaller than the count variation produced by the ICRH when NBI is active as well. The PHS, plotted in Fig. 9, again normalised to each other at channels 50-100, show that the high energy tail is slightly more pronounced when the ICRH is switched on (green) compared to the pure NBI phase (red). 
The zoomed plot, Fig. 9 (b), shows the range of the energetic tail, around channels 160-210, i.e. 20-70 channels above the estimated $2.45 \mathrm{MeV}$ reference (channel 140), corresponding roughly to $\sim 3 \mathrm{MeV}$ neutron energy. The effect on the PHS, though, is rather small, albeit beyond statistical uncertainties. Further experiments are needed to assess a possible trend.

Finally, some qualitative gamma spectroscopy is possible as well. In the
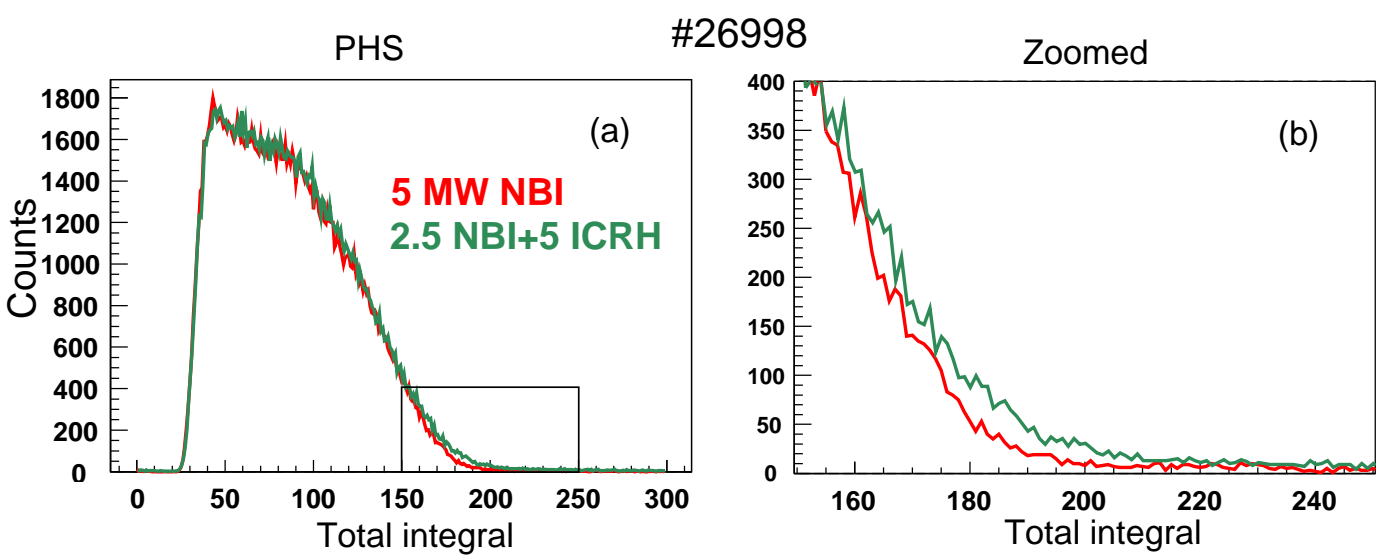

Figure 9: PHS for discharge \# 26998 in a phase with $P_{N B I}=5 \mathrm{MW}, P_{I C R H}$ $=0$ (red) and $P_{N B I}=2.5 \mathrm{MW}, P_{I C R H}=5 \mathrm{MW}$ (green). (b) is a zoom of the same picture around channels 150-250. The ICRH phase has a PHS with higher tail at high energy.

disrupting discharge \# 26908, for instance, gamma rays up to $1.5 \mathrm{MeV}$ were detected during the disruption. The energy is estimated with a reference PHS obtained with a ${ }^{207} \mathrm{Bi}$ source, emitting lines at $0.569,1.064$ and $1.771 \mathrm{MeV}$. Both gamma PHS of the ASDEX Upgrade discharge \# 26908 at disruption time and of a reference ${ }^{207} \mathrm{Bi}$ acquisition are shown in Fig. 10.

\section{Conclusions}

A CNS based on the BC501A liquid scintillator has been successfully installed on the ASDEX Upgrade tokamak. Thanks to the digital acquisition system, the high pulse statistics enables accurate PHS over a time interval of the order of $100 \mathrm{~ms}$, comparable to the energy confinement time and to the NBI ions slowing down time of ASDEX Upgrade. A flexible and fast DPSD 


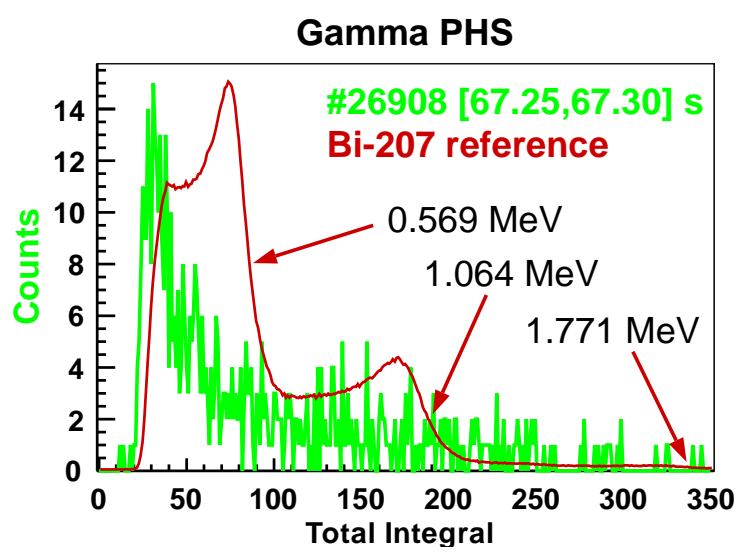

Figure 10: Gamma PHS of a disrupting discharge \# 26908 (green) and of a reference ${ }^{207} \mathrm{Bi}$ acquisition (red). Gamma energies around $1 \mathrm{MeV}$ are observed during the disruptive phase.

package, based on $\mathrm{C}++/ \mathrm{ROOT}$, has been developed for gamma-neutron discrimination and pulse integration. Burn-up d-t neutrons are observed as well. First PHS analysis exhibits the expected behaviour, giving confidence in the quality of the measurements. The time behaviour of the reference ${ }^{238} \mathrm{U}$ fission chamber is well reproduced. Different rates are observed depending on the NB injection angle: for the same plasma parameters, parallel injection yields less neutrons than perpendicular injection, so that the cross-calibration factor with respect to the reference ${ }^{238} \mathrm{U}$ chamber is not the same in the two cases. This is a consequence of the kinematics of the D-d fusion reaction in the laboratory frame. The PHS hints to a broader energy distribution for perpendicular injection, which is expected due to the detector's line of sight, also perpendicular to the plasma's magnetic surfaces. The signature of the synergy between NBI and ICRH is also shown by the synergetically increased count rate and the slightly more pronounced tail of the PHS at high energies. Future experiments with pure ICRH are expected to increase the statistical evidence and to improve our knowledge of the modified fast ions distribution in presence of NBI and ICRH simultaneously. Some qualitative gamma analysis can be performed with the CNS in order to better diagnose runaway electrons in disruptive plasma discharges.

A natural extension of the present work is of course the deconvolution of the PHS into energy neutron distribution functions $f\left(E_{n}\right)$. A code based on 
maximum entropy will be used [16][17]. The experimental campaign for the detector characterisation at the PTB facility is already accomplished, but the data have still to be evaluated. On the simulation side, a forward prediction of $f\left(E_{n}\right)$ is foreseen by coupling the MonteCarlo fast ions code NUBEAM [18] with a neutron spectrum code such as GENESIS [19].

Further experiments are envisaged to exploit the CNS: discharges with pure ICRH are required as a reference to tackle the synergies between NBI and ICRH at the level of the fast ions distribution function, a footprint of $f\left(E_{n}\right)$. Long ohmic phases as well as separated $60 \mathrm{kV}$ and $93 \mathrm{kV}$ NBI phases will help to assess experimentally the extent of the CNS energy resolution.

Future enhancements concern a faster data transmission, in order to improve the time resolution to resolve a single NBI slowing down time interval as well as the plasma energy confinement time.

\section{References}

[1] M. Garcia-Munoz et al., Nuclear Fusion 51 (2011) 103013

[2] R. B. White and M. S. Chance, Phys. Fluids 27 (1984) 2455

[3] B. Geiger et al., Plasma Physics and Controlled Fusion 53 (2011) 065010

[4] C. Höhbauer, IPP Report 1/339

[5] A. Zimbal et al., Rev. Sci. Instrum. 75 (2004) 3553

[6] L. Giacomelli et al., Rev. Sci. Instrum. 182 (2011) 123504

[7] B. Esposito et al., Nucl. Instr. and Methods in Physics Research A 572 (2007) 355

[8] M. Riva et al., Fusion Eng. Des. 82 (2007) 1245

[9] V. Krasilnikov et al., Comp. Phys. Comm. 82 (2011) 735

[10] D. Marocco et al., IEEE Trans. Nucl. Sci. 56 (2009) 1168

[11] F. Belli et al., Nucl. Instrum. Meth. Phys. Res. A 595 (2008) 512

[12] H. Klein, S. Neumann, Nuclear Instruments and Methods in Physics Research A476 (2002) 132 
[13] H. Kimura et al., Nuclear Fusion 31 (1991) 83

[14] R. Bilato et al., Nuclear Fusion 51 (2011) 103034

[15] A. V. Krasilnikov et al., Plasma Phys. Control. Fusion 51 (2009) 044005

[16] M. Reginatto, A. Zimbal, Rev. Sci. Instrum. 79 (2008) 023505

[17] F. Gagnon-Moisan et al., "Results for the response function determination of the Compact Neutron Spectrometer", this conference

[18] A. Pankin et al., D. McCune, R. Andre et al., Comp. Phys. Comm. 159, No. 3 (2004) 157

[19] M. Tardocchi et al., Phys. Rev. Lett. 107 (2011) 205002 